\title{
Regenerative Nd:glass amplifier seeded with a Nd:fiber laser
}

\author{
M. Hofer, M. H. Ober, F. Haberl, and M. E. Fermann* \\ Technische Universität Wien, Abteilung Quantenelektronik und Lasertechnik, Gusshausstrasse 27/359/9, A-1040 Vienna, Austria
}

E. R. Taylor and K. P. Jedrzejewski

Optoelectronics Research Centre, Southampton University, Southampton SOg $5 \mathrm{NH}, \mathrm{UK}$

Received December 24, 1991

Wavelength tuning and broad-bandwidth operation of a passively mode-locked $\mathrm{Nd}$ : fiber laser is demonstrated at $1060 \mu \mathrm{m}$. The oscillator pulses are used to seed a bulk regenerative Nd:glass amplifier, and $300-\mathrm{fs}$ transformlimited pulses with an energy of $10 \mu \mathrm{J}$ are obtained after 31 round trips at a repetition rate of $500 \mathrm{~Hz}$.

Passively mode-locked rare-earth-doped fiber lasers are a well-established medium for the generation of ultrashort pulses, ${ }^{1-3}$ allowing one to generate pulses with a bandwidth as large as the bandwidth of the gain medium. ${ }^{4}$ In particular, Nd-doped fiber oscillators are of special interest because the oscillator pulses may be used in bulk-medium or high-power $\mathrm{Nd}$ :glass amplifier systems. ${ }^{5-7}$ In these applications, Nd:phosphate glass with a peak emission wavelength at $1.053 \mu \mathrm{m}$ is preferred owing to its good thermal properties and low nonlinear refractive index. However, high-quality Nd:phosphate or fluoride fibers (with a similar peak emission wavelength) do not exist yet, and typical low-loss leadglass or silica fibers emit at $\sim 1.06 \mu \mathrm{m}$. We show here that in a highly nonlinear Nd:fiber oscillator wavelength tuning down to the preferred wavelength range is possible, or, alternatively, broad-bandwidth operation of these oscillators may be initiated, where the preferred spectral range may be selected by extracavity spectral filtering. Broad-bandwidth operation is obtained with strongly chirped pulses, which also allows the maximization of the oscillator pulse energy. The generated short oscillator pulses allow one to take full advantage of the available bandwidth in a bulk Nd:glass amplifier and the generation of subpicosecond transform-limited pulses with microjoule energies.

We used a lead-glass fiber consisting of a Schott F2/F 7 glass combination doped with $\approx 3000$ parts in $10^{6}$ of $\mathrm{Nd}^{3+}$ as the oscillator in our experiments. The peak emission wavelength is $1.060 \mu \mathrm{m}$, and the nonlinear refractive index $\left(n_{2}=10^{-19} \mathrm{~m}^{2} / \mathrm{W}\right)$ and the positive group-velocity dispersion (GVD) $\left(\boldsymbol{\beta}_{2}=\right.$ $70,000 \mathrm{fs}^{2} / \mathrm{m}$ ) of this material are approximately three times higher than those in a silica glass. The fiber length was $23 \mathrm{~cm}$, and the core diameter was $5 \mu \mathrm{m}$. The fiber was pumped with a mixture of 752- and 799-nm beams from a krypton laser with absorbed pump powers of as much as $180 \mathrm{~mW}$. The absorption length was $\approx 10 \mathrm{~cm}$. Intracavity dispersion adjustment was achieved by using a single Brewster-cut $\mathrm{ZnS}$ prism pair, which provided a negative GVD of $57,000 \mathrm{fs}^{2} / \mathrm{m}$. Passive amplitude modulation in the cavity was obtained with nonlinear-polarization evolution, ${ }^{2,4}$ where passive mode locking was initiated with an acousto-optic modulator, which was switched off once pulses were generated. With the proper polarization setting, the oscillator remained stable for hours and required only small adjustments on a day-to-day basis. The oscillator cavity was thus of similar design as that in Ref. 2 . In addition, a variable aperture was translated in front of the output coupler for spectral windowing.

With a $20 \%$ output coupler, we obtained a cw lasing threshold of $<5 \mathrm{~mW}$ of absorbed pump power, a maximum lasing power of $20 \mathrm{~mW}$, and a slope efficiency of $11 \%$. With a $50 \%$ output coupler, the slope efficiency and the maxium lasing power were higher by a factor of 2 . The maximum $\mathrm{cw}$ mode-locked power was $12 \mathrm{~mW}$, where the pulse repetition rate was $100 \mathrm{MHz}$. A minimum pulse width of $150 \mathrm{fs}$ with a time-bandwidth product of 0.5 for a sech ${ }^{2}$ shape was produced with an overall cavity dispersion of approximately $-20,000 \mathrm{fs}^{2}$. However, pulse breakup (leading to higher-order mode locking or pulse bunching) was observed to occur for cw modelocked powers as low as $3 \mathrm{~mW}$. At an overall cavity dispersion of approximately $-40,000 \mathrm{fs}^{2}$, wavelength tuning was performable, where $\operatorname{sech}^{2}$-shaped pulses with a time--bandwidth product of 0.8 were obtained in the spectral range from 1.053 to $1.070 \mu \mathrm{m}$. A pulse spectrum at the lower end of the tuning range is shown in Fig. 1, which also demonstrates the complete elimination of a $\mathrm{cw}$ background. However, because the adjustment of the cavity parameters required to produce a large spectral shift was critical, for experimental convenience the amplifier seed pulses were produced by using a different approach. In this procedure, the pulses were generated with more positive values of GVD. Typically, as the cavity dispersion is increased to more positive values, the pulse bandwidth increases equally, resulting in increasingly strongly chirped pulse solutions. ${ }^{4}$ The pulse bandwidth may even exceed the bandwidth of 


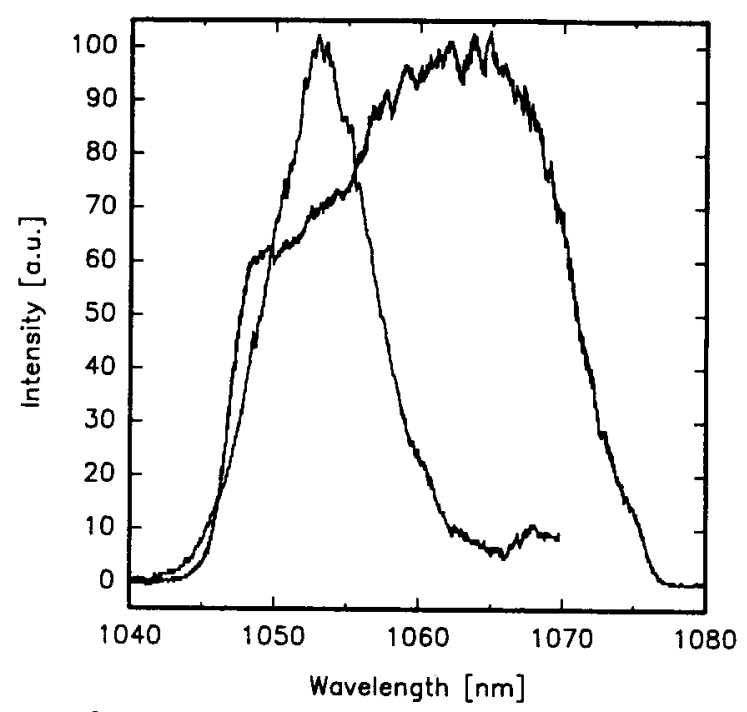

Fig. 1. Spectrally shifted and superbroad oscillator pulse spectrum.

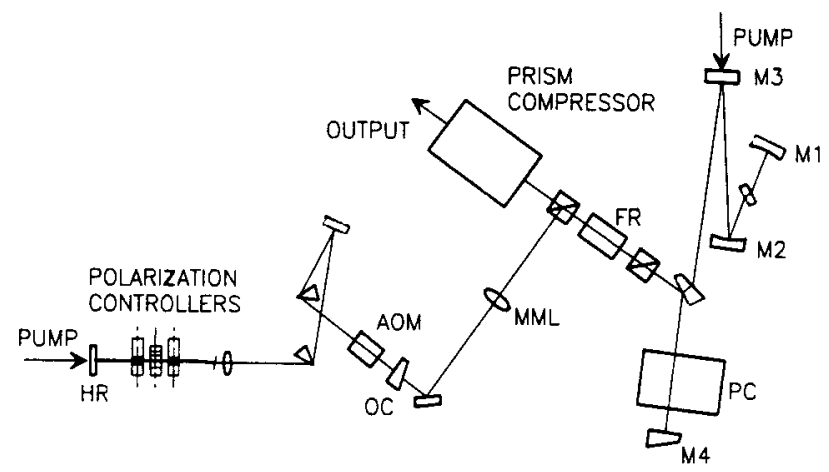

Fig. 2. Experimental arrangement of the oscillator (mirrors $\mathrm{HR}-\mathrm{OC}$ ) and amplifier (mirrors M1-M4) system. $\mathrm{HR}$, high reflectors; AOM, acousto-optic modulator; OC, output coupler; MML, mode-matching lens; FR, Faraday rotator; PC, Pockels cell.

the gain medium for high-power pulses. ${ }^{4}$ Here we employed a GVD of $\approx 0 \mathrm{fs}^{2}$ in the cavity such that the generated pulse spectrum was sufficiently broad to cover the wavelength region of interest (see Fig. 1). The corresponding FWHM oscillator pulse width was $530 \mathrm{fs}$, which gave a time-bandwidth product of 3.4. The pulses could be compressed externally to within a factor of 3 of the bandwidth limit, and pulse compression down to the bandwidth limit required further spectral filtering.

An additional feature of these strongly chirped gain-guided pulse solutions is that their energy content can be significantly higher than is possible for pulses in the negative GVD regime, and pulse breakup was not observed even for the highest $\mathrm{cw}$ mode-locked powers. The reason may be understood from approximate analytical solutions of the modelocked pulses in the cavity, ${ }^{4,8}$ where all cavity parameters are assumed to be evenly distributed along the fiber length. The corresponding lengthaveraged propagation equation has a chirped sech-pulse solution. The fiber system is well approximated by this type of equation, particularly for small prism separations, because the variation in pulse width along the cavity also becomes small then. ${ }^{4}$ High-energy pulse solutions in the positive GVD regime result in strongly chirped pulses with small peak powers, whereas low-energy pulse solutions do not exist. On the other hand, in the negative GVD regime, high-energy pulse solutions are weakly chirped and have correspondingly high peak powers. In addition, low-energy pulse solutions with an even lower chirp also exist. In the presence of pulse perturbations and a saturation of the passive amplitude modulation in the cavity, high-energy pulses therefore tend to break up into pulse bunches or pulses at multiples of the cavity round-trip frequency.

The complete oscillator-amplifier system is shown in Fig. 2. Here, the bulk gain medium is a phosphate glass plate of 6-mm length and 1-mm thickness doped with 2 wt.\% Nd. To ensure maximum long-term stability, the amplifier was $\mathrm{cw}$ pumped with only $0.7 \mathrm{~W}$ from the krypton laser, well below a possible pumping level of $1.6 \mathrm{~W}$ determined from thermal considerations. The pump beam underfilled the laser beam waist radius of $30 \mu \mathrm{m}$ obtained by using two focusing mirrors with $150-\mathrm{mm}$ radii of curvature. Amplification of single pulses from the
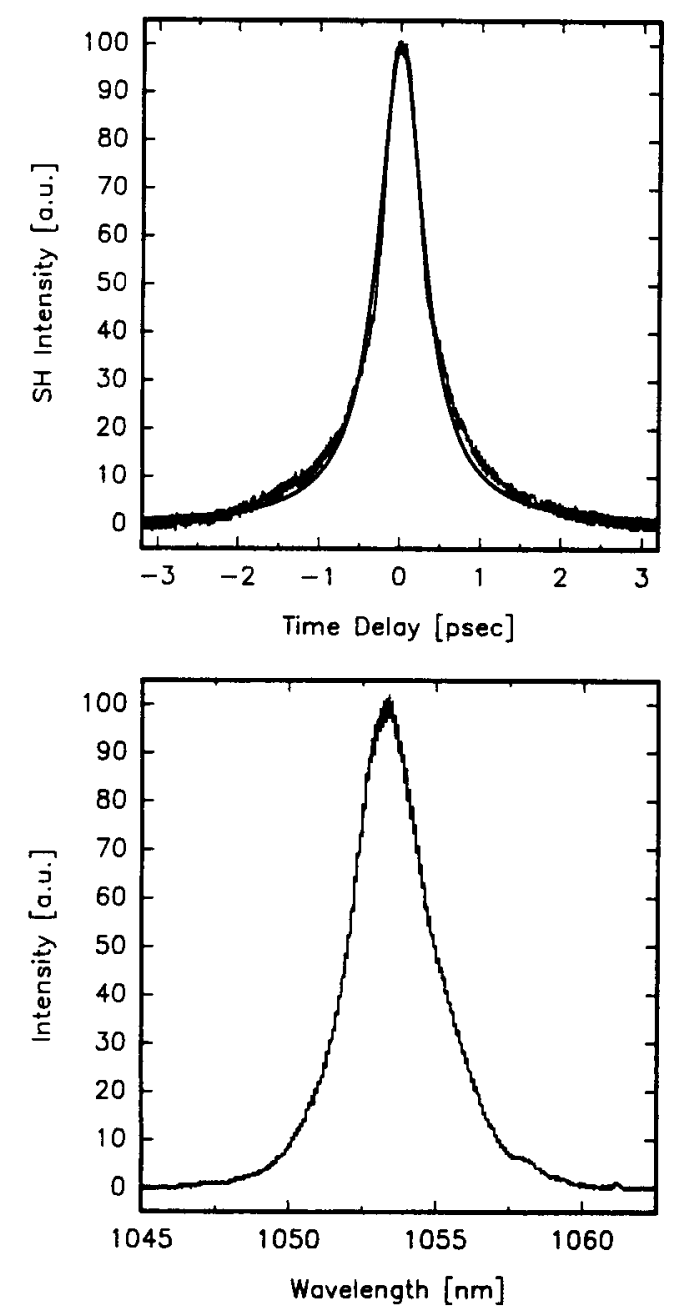

Fig. 3. Background-free autocorrelation of amplified pulses (top) and the corresponding pulse spectrum (bottom). Also shown is a Lorentzian fit to the autocorrelation trace (solid curve in top graph). 
oscillator was ensured by insertion of a Pockels cell with a static $\lambda / 4$ phase offset into the amplifier cavity. The prevention of feedback into the oscillator was critical and avoided by a polarizer-Faraday rotator arrangement, which also served to channel the amplified pulses out of the system. After amplification, the pulses were compressed to their minimal pulse width by a prism pair arrangement.

The oscillator pulse energy was $120 \mathrm{pJ}$. With mode matching and spectral losses taken into account, the final seed pulse energy into the amplifier was $10 \mathrm{pJ}$. The typical round-trip amplifier gain was 1.5. Saturation occurred after 36 round trips in the amplifier, where the pulse energy was $13 \mu \mathrm{J}$ and the typical uncompressed pulse width was 3 ps (assuming a sech ${ }^{2}$ shape). At this point, the accumulated nonlinear phase delay for the 3-ps pulses in the amplifier was $\approx 1.6 \pi$, which led to the onset of self-focusing and noncompressible spectral broadening of the amplified pulses. By limiting the number of round trips to 31 and the amplified pulse energy to $10 \mu \mathrm{J}$, thus also operating the amplifier slightly away from saturation, nonlinear spectral broadening in the amplifier was sufficiently reduced. After compression, the resulting pulse energy was $8 \mu \mathrm{J}$.

An autocorrelation trace of the compressed amplified pulse and the pulse spectrum are shown in Fig. 3. The amplified pulses had a Lorentzian shape with a FWHM width of $300 \mathrm{fs}$. The timebandwidth product was 0.24 , within $10 \%$ of the bandwidth limit of 0.22 . To our knowledge, these are the shortest pulses yet produced from an all-glass oscillator-amplifier system at this energy level. Because the oscillator may also be pumped with a laser diode, the system may be upgraded to a diode-pumped all-solid-state version that produces subpicosecond pulses with energies up to the millijoule level.
In summary, we have demonstrated what is to our knowledge the first passive mode locking in a highly nonlinear lead-glass fiber. By using strongly chirped oscillator pulses, self-phase modulation in the oscillator was reduced and the energy content of the oscillator pulses was optimized. The oscillator pulses were of sufficient quality to allow seeding of a bulk Nd:glass amplifier system. We are currently investigating whether strongly chirped oscillator pulses may be used to reduce self-phase modulation in the amplifier further.

We are indebted to T. Brabec, F. Krausz, G. Mourou, A. J. Schmidt, C. Spielmann, and E. Wintner for stimulating discussions. This research was supported by Fonds zur Förderung der wissenschaflichen Forschung in Österreich (projects P8024 and P8391) and by the Österreichische Nationalbank (project 4049).

*Present address, Bell Communications Research, 331 Newman Springs Road, Red Bank, New Jersey 07701.

\section{References}

1. M. E. Fermann, M. Hofer, F. Haberl, A. J. Schmidt, and L. Turi, Opt. Lett. 16, 244 (1991).

2. M. Hofer, M. E. Fermann, F. Haberl, M. H. Ober, and A. J. Schmidt, Opt. Lett. 16, 503 (1991).

3. I. N. Duling III, Opt. Lett. 16, 539 (1991).

4. M. Hofer, M. H. Ober, F. Haberl, and M. E. Fermann, IEEE J. Quantum Electron. 28, 720 (1992).

5. L. Yan, P. T. Ho, C. H. Lee, and G. L. Burdge, IEEE J. Quantum Electron. 25, 2341 (1989).

6. C. Sauteret, D. Husson, G. Thiell, S. Seznec, S. Gary, A. Migus, and G. Mourou, Opt. Lett. 16, 238 (1991).

7. F. G. Patterson, R. Gonzales, and M. D. Perry, Opt. Lett. 16, 1707 (1991).

8. P. A. Belanger, L. Gagnon, and C. Paré, Opt. Lett. 14, 943 (1989). 